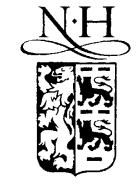

ELSEVIER
Available at

www.ComputerScienceWeb.com

POWERED BY SCIENCE (d) DRECT

Pattern Recognition Letters 24 (2003) 1349-1360
Pattern Recognition Letters

www.elsevier.com/locate/patrec

\title{
Image enhancement and minutiae matching in fingerprint verification
}

\author{
Yuliang $\mathrm{He}^{*}$, Jie Tian *, Xiping Luo, Tanghui Zhang \\ Fingerpass Research Group, Artificial Intelligent Laboratory, Institute of Automation, Chinese Academy of Sciences, \\ P.O. Box 2728, 100080, HaiDian District, Beijing, China
}

Received 5 April 2002; received in revised form 27 October 2002

\begin{abstract}
Fingerprint image enhancement and minutiae matching are two key steps in an automatic fingerprint identification system. In this paper, we develop a fingerprint image enhancement algorithm based on orientation fields; According to the principles of Jain et al.'s matching algorithm, we also introduce ideas along the following three aspects: introduction of ridge information into the minutiae matching process in a simple but effective way, which solves the problem of reference point pair selection with low computational cost; use of a variable sized bounding box to make our algorithm more robust to non-linear deformation between fingerprint images; use of a simpler alignment method in our algorithm. Experiments using the Fingerprint Verification Competition 2000 (FVC2000) databases with the FVC2000 performance evaluation show that these ideas are effective.
\end{abstract}

(C) 2002 Elsevier Science B.V. All rights reserved.

Keywords: Fingerprint; Image enhancement; Minutiae matching and orientation field

\section{Introduction}

Automatic fingerprint identification systems (AFIS) have been widely used. An AFIS consists of fingerprint classification, fingerprint image enhancement and fingerprint matching. In this study, we develop some methods for fingerprint image enhancement and fingerprint matching.

Our system (Fig. 1) consists of two phases: offline and on-line. In the off-line phase, a fingerprint

\footnotetext{
${ }^{*}$ Corresponding authors. Tel.: +86-10-82618465; fax: +8610-85191212 (Y. He). Tel.: +86-10-62559951; fax: +86-1062522105 (J. Tian).

E-mail address: tian@doctor.com (J. Tian).
}

is acquired, enhanced using different algorithms, where after features of the fingerprint are extracted and stored in a database as a template. In the online phase, a fingerprint is acquired, enhanced and features of the fingerprint are extracted, fed to a matching model and matched against template models in the database.

In practice, a fingerprint may be corrupted by noise due to impression, skin, reader etc., during image capture. Fingerprint enhancement is used to recover the topology structure of ridges and valleys from the noisy image. Most of the fingerprint enhancement algorithms are based on the estimation of the orientation field (Huang, 1993; Jain et al., 1997; Lin et al., 1998). In this study, we 


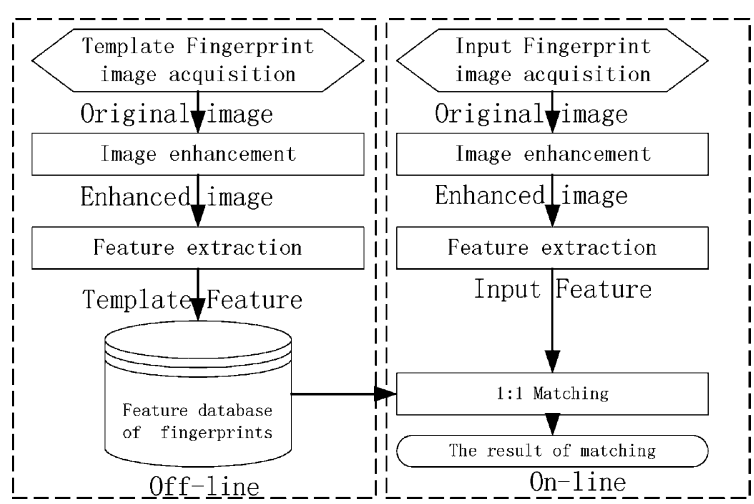

Fig. 1. Flowchart of our system.

develop a fingerprint image enhancement algorithm based on orientation fields.

In the past years, efforts have been made in fingerprint matching which is a key step in AFIS. The most prevalent model for AFIS is the minutiae-coordinate model. The two most prominent structures are ridge endings and ridge bifurcations, which are usually called minutiae. By representing the minutiae as a point pattern, the automatic fingerprint verification can be reduced to a point pattern model-matching (minutiae matching) problem. A number of point pattern matching algorithms have been proposed in the literature, such as the relaxation approach (Ranade and Rosenfeld, 1980), the fast algorithm based on 2-D clusters (Chang et al., 1997), the triangular matching and dynamic time warping approach (Miklós and Kovács-Vajna, 2000) and the local and global structure matching approach (Jiang and Yau, 2000). Jain et al. (1997) proposed an algorithm to deal with the problem of point pattern matching in fingerprint recognition by changing the points to the polar coordinate system and comparing the point sets using a string matching algorithm.

The minutiae-matching algorithm in this paper originates from the algorithm of Jain et al. (1997). But our algorithm differs in three aspects. Firstly, the alignment method we use is different. Secondly, unlike the method proposed by Jain et al. (1997), which uses ridge information only in the alignment stage, we introduce ridge information into the matching process. Finally, the method of Jain et al. uses a fixed sized bounding box during the matching process, whereas we replace it with a variable bounding box, which is more robust to non-linear deformations between two fingerprints.

In the following sections, we discuss our method in greater details.

\section{Fingerprint image enhancement}

\subsection{Estimation of orientation field}

In general, a fingerprint has a well-defined orientation field. The estimation of the orientation field is critical to fingerprint image enhancement. To estimate the orientation field, we divide the ridge direction of a pixel into 8 directions (Fig. 2).

To decide the ridge direction of each pixel in the image, we compute the average grey value in direction $i(i=0,1, \ldots, 7$ means one of the 8 directions) in a $9 \times 9$ window with the pixel as the centre. In other words, we compute the average grey value of the pixels labelled " $i$ " (Fig. 2) and obtained $G[i]$. The 8 mean grey values are divided into 4 groups with the two directions in each group perpendicular to each other. Group $j(j=0,1$, $2,3)$ contains direction $j$ and $j+4$. The absolute value of the difference of the mean grey value is calculated in each group as:

$G_{d}[j]=|G[j]-G[j+4]| \quad(j=0,1,2,3)$

Set the two directions in the group with the largest difference value as possible ridge directions. If

$i_{\text {Max }}=\arg \left\{\operatorname{Max}_{i \in\{0,1,2,3\}}\left(G_{d}(i)\right)\right\}$

Fig. 2. Eight ridge directions of a pixel. 
then $i_{\text {Max }}$ and $i_{\text {Max }}+4$ are possible ridge directions. The ridge direction in the pixel is decided by

$D_{i}= \begin{cases}i_{\text {Max }} & \text { if }\left(\mid \text { Grey }-G\left[i_{\text {Max }}\right] \mid\right. \\ & \left.<\mid \text { Grey }-G\left[i_{\text {Max }}+4\right] \mid\right) \\ i_{\text {Max }}+4 & \text { otherwise }\end{cases}$

where Grey is the grey value at this pixel.

To reduce the effect of noise, we partition the image into small blocks of size $16 \times 16$ and set the ridge direction of each pixel in one block as the direction of that block, that is, the mean direction of all the pixels in the block. To obtain the mean direction of a block, we calculate the number of pixels in the block where ridge direction is estimated as $i(i=0,1, \ldots, 7)$ and set this number as $N_{i}$. The mean direction of the block is:

$$
B=\arg \left\{\operatorname{Max}_{i=\{0,1, \ldots, 7\}}\left(N_{i}\right)\right\}
$$

\subsection{Binarization and thinning}

In our AFIS, we extracted minutiae of a fingerprint from its thinned image. The grey values in raw images were of a continuous slope, indicating that binarization by means of a global threshold technique is unsuitable for further processing. To solve the problem, we develop a method to directly binarize the image with its orientation field, which is fast to obtain binary image.

In a pixel with ridge direction $i$, we calculate the mean grey value in direction $i$ and in the direction perpendicular to $i$, i.e. direction $i_{\mathrm{Ver}}=i+4$ to obtain $G[i]$ and $G\left[i_{\mathrm{Ver}}\right]$, then to binarize the pixel as:

$i_{\text {Val }}= \begin{cases}255 & \text { if } G[i] \geqslant G\left[i_{\mathrm{Ver}}\right] \\ 0 & \text { otherwise }\end{cases}$

where $i_{\mathrm{Val}}$ is the grey value of the binarized image in this pixel. Grey value 255 denotes the background of the image and the valley of the fingerprint. Grey value 0 denotes the ridge of the fingerprint in the image.

We thin the binary image of a fingerprint with the thinning method introduced in (Naccache and
Shinghal, 1984) and obtained the skeleton of the fingerprint. It is more accurate and easier to detect its minutiae and to trace its ridges from a thinning image than directly from the raw image.

Since a fingerprint may be corrupted by noise due to impression, skin, reader, etc. during image capture, post-processing should be done (Luo and Tian, 2000).

\subsection{Results of image enhancement}

The performance of the enhancement algorithm was evaluated on various fingerprint images, including the four databases of the Fingerprint Verification Competition 2000 (FVC2000). Figs. 3-6 are four typical examples of the enhancement performance. Figs. 3(a), 4(a), 5(a) and 6(a) are selected randomly from the four fingerprint databases of FVC2000 respectively. Figs. 3(b), 4(b), 5(b) and 6(b) are their orientation field images. Figs. 3(c), 4(c), 5(c) and 6(c) are their binary images. Figs. 3(d), 4(d), 5(d) and 6(d) are their thinned images. Their average times of enhancement are $0.41,0.34,0.91$ and $0.26 \mathrm{~s}$ respectively; experiments on enhancement were conducted on a Pentium III450 MHz machine using windows2000.

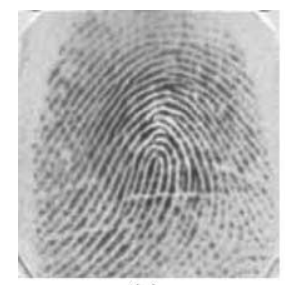

(a)

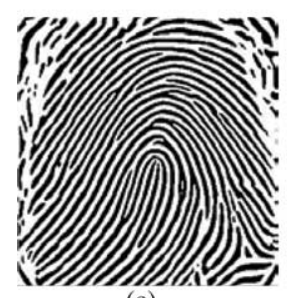

(c)

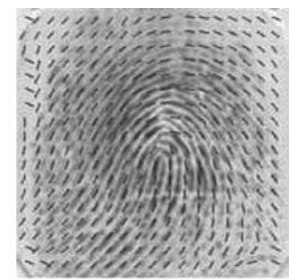

(b)

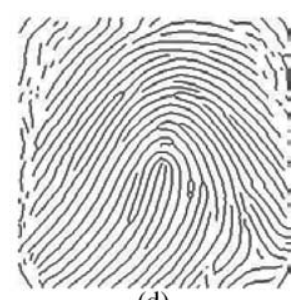

(d)
Fig. 3. A $300 \times 300$ fingerprint image of DB1_a of FVC2000 and its enhancement results. 


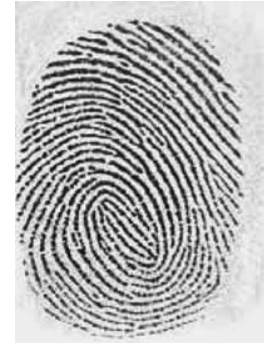

(a)

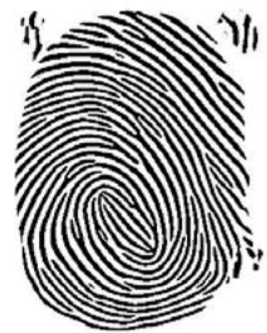

(c)

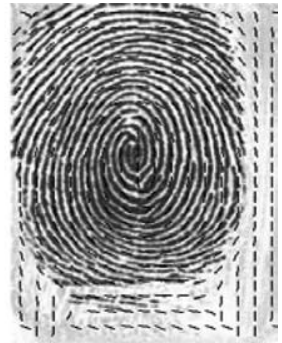

(b)

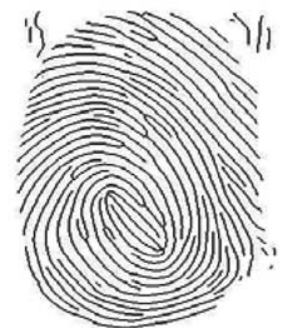

(d)
Fig. 4. A $256 \times 364$ fingerprint image of DB2_a of FVC2000 and its enhancement results.

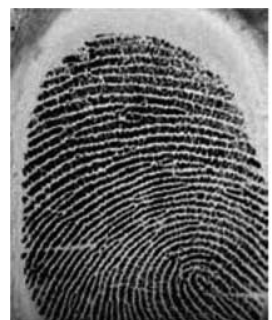

(a)

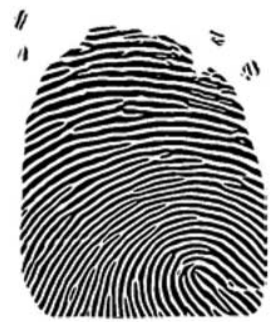

(c)

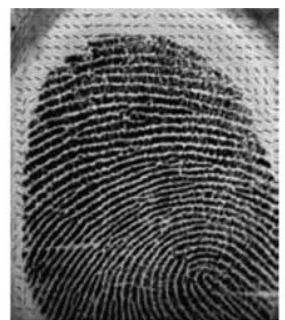

(b)

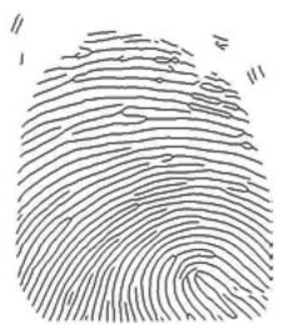

(d)
Fig. 5. A $448 \times 478$ fingerprint image of DB3_a of FVC2000 and its enhancement results.

The results show that the method of enhancement is faster than other methods (Huang, 1993; Jain et al., 1997; Lin et al., 1998).

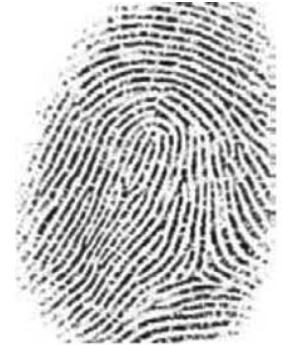

(a)

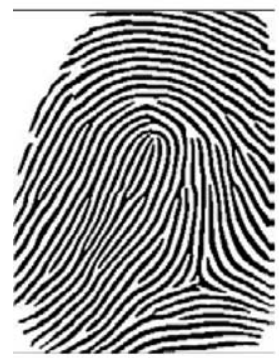

(c)

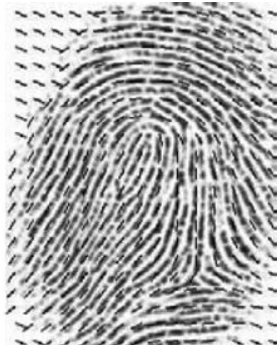

(b)

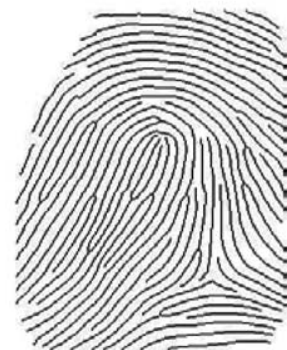

(d)
Fig. 6. A $240 \times 300$ fingerprint image of DB4_a of FVC2000 and its results enhancement.

\section{Minutiae extraction}

We extract the minutiae of a fingerprint from its thinned image (Farina et al., 1999; Ratha et al., 1995; Xiao and Raafat, 1991). Let $m(F)$ be the number of minutia points in a fingerprint $F$. The set of all the minutia information is $M(F)=$ $\left\{M(F)(i)=\left\{x_{i}, y_{i}, \theta_{i}, t_{i}, d_{i n}, \alpha_{i n} ; n=1,2, \ldots, L\right\}^{\mathrm{T}}\right.$ $i=1,2, \ldots, m(F)\}$ where the minutia $M(F)(i)$ denotes the $i$ th minutia in the minutiae set $M(F)$. The features of the minutia $M(F)(i)$ are as follows:

(1) $x_{i}$, and $y_{i}$ denote its coordinates.

(2) $\theta_{i}$ denotes its orientation, the angle from OX to its local ridge direction at $M(F)(i)$ in the anticlockwise direction.

(3) $t_{i}$ denotes the type of the minutia point, which is either ending or bifurcation.

(4) $\left(d_{i n}, \alpha_{i n} ; n=1,2, \ldots, L, L \leqslant 4\right)$ denotes the associated ridge corresponding to $M(F)(i)$. The associated ridge is represented by points $N_{i n}$ $(n=1,2, \ldots, L)$ sampled at the average interridge distance along the ridge linked with $M(F)(i)$. In our algorithm, at least 4 points are recorded on an associated ridge. $d_{i n}$ 


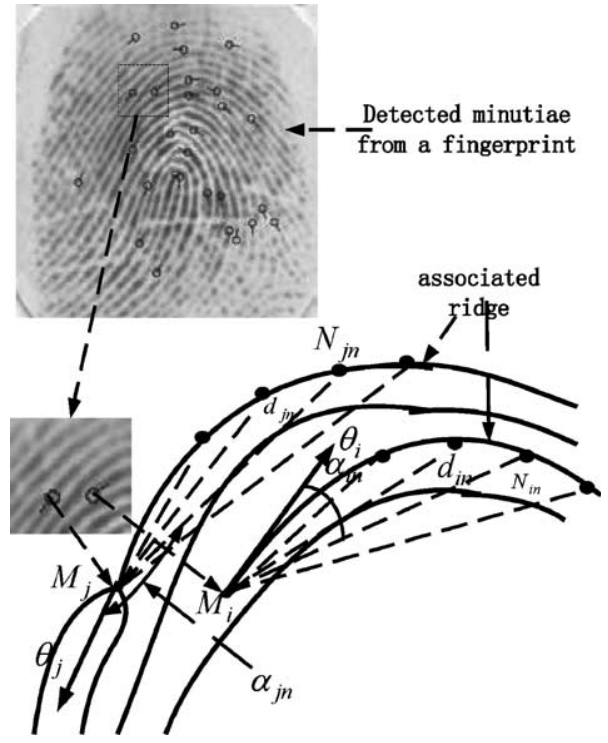

Fig. 7. Minutiae from a fingerprint and their associated ridges.

$(n=1,2, \ldots, L)$ denotes the distance between the minutia $M(F)(i)$ and $N_{i n} ; \alpha_{i n}$ denotes the angle between the direction from the minutia $M(F)(i)$ to $N_{i n}$ and the direction $\theta_{i}$.

Fig. 7 shows the minutiae of a fingerprint and their associated ridges. For the bifurcation ridge $M(F)(j)$, the associated ridge is represented with the ridge which is the closest to the reverse of $\theta_{i}$. For ridge ending $M(F)(i)$, the associated ridge is just the ridge linked with $M(F)(i)$. In minutiae matching, the associated ridge is used to align the two sets of planar point patterns to be matched. Furthermore, the rotation angle between any pair of ridges in the two minutiae point sets, are used to decide whether the two minutiae associated with this pair of ridges can be taken as the corresponding minutiae pair.

$M(F)$ determines the global minutia structure of the fingerprint $F$. For convenience, let $x(F, i)$ denote $x_{i}$ in minutia $M(F)(i)$ of fingerprint $F, y(F, i)$ for $y_{i}, \theta(F, i)$ for $\theta_{i}, t(F, i)$ for $t_{i}, d\left(F, i_{n}\right)(n=1$, $2, \ldots, L)$ for $d_{i n}, a\left(F, i_{n}\right)(n=1,2, \ldots, L)$ for $\alpha_{i n}$.

\section{Minutiae matching}

Minutiae-matching in the polar coordinate system has several advantages (Jain et al., 1997).

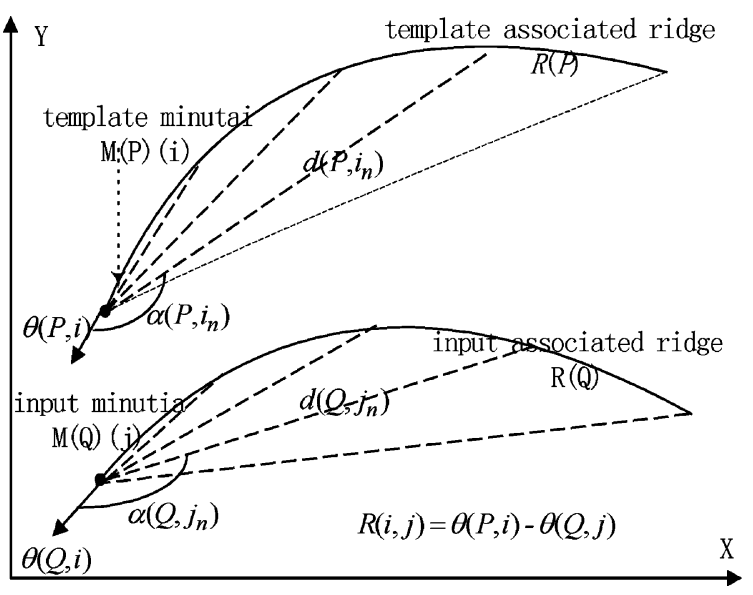

Fig. 8. Alignment of input and template associated ridges.

The non-linear deformation of fingerprints has a radial property, which radiates outward from a certain region. Therefore, it is beneficial to model the minutiae set in the polar space, without consideration of the translation between the input and template images in polar coordinates (Fig. 8). The translation can be compensated in the polar coordinate system because it is the same translation between the other corresponding point pairs as between the reference point pair. At the same time, it is much easier to handle rotation in the polar space than in the Cartesian space. Hence, we do minutiae matching in the polar coordinate system.

Even if an input image comes from the same finger as the template image in the database, there are transformations such as translation, rotation and scaling. When the minutiae of an input fingerprint are matched to the minutiae of the template fingerprint in the database, we must estimate these parameters to align input minutiae with the template minutiae. We assume that the scaling factor between input and template images is identical since both images are captured with the same device. In addition, we do not need the translation between input and template images in the polar coordinates, except the rotation parameter between input and template images.

The existence of non-linear deformation of fingerprints, an inherent property of fingerprint impression, prevents exact recovery or the position 
of each input minutia with respect to its corresponding minutia in the template. Therefore, the matching algorithm should be elastic, or capable of tolerating, to some extent, the deformations due to inexact extraction of minutia positions and nonlinear deformations. Jain and coworkers (Maio et al., 2002) dealt with the problem of elastic matching by placing a bounding box around each template minutia, which specifies all the possible positions of the corresponding input minutia with respect to the template minutia and restricts the corresponding minutia in the image to be contained in this box. The method (Jain et al., 1997) is more effective by adjusting the bounding box when an inexact match is found during the matching process, showing an ability to compensate for minutia localization error and non-linear deformation. The method (Jain et al., 1997) needs further modification by using variable sized bounding boxes to be more robust to non-linear deformations between two fingerprint images.

\subsection{Alignment of minutiae set}

$P$ and $M(P)$ denote a template fingerprint and its minutiae set respectively. $Q$ and $M(Q)$ denote the input fingerprint and its minutiae set respectively.

To change the minutiae to the polar coordinate system, we choose a pair of minutiae in the template minutiae set and the input minutiae set as reference points (the origin of the polar coordinate systems) and get the polar coordinates of other minutiae according to the reference points. Since we know nothing about the correspondence of template and input point sets, we will considered each possible pair of reference points.

For each minutia $M(P)(i)(1 \leqslant i \leqslant m(P))$ in the template minutiae set and each minutia $M(Q)(j)$ $(1 \leqslant j \leqslant m(Q))$ in the input minutiae set, let $R(i, j)$ denote the rotation angle between input and the template images if we take $M(P)(i)$ and $M(Q)(j)$ as the reference points of the corresponding image. If $M(P)(i)$ and $M(Q)(j)$ are considered as a pair of corresponding minutiae, or the associated ridges of $M(P)(i)$ and $M(Q)(j)$ are similar to each other in a certain degree, $R(i, j) \in\{0,1, \ldots, 359\}$. Otherwise
$R(i, j)=400$, which indicates that $M(P)(i)$ and $M(Q)(j)$ can not be considered as a pair of corresponding minutiae.

If $M(P)(i)$ and $M(Q)(j)$ are of different types, one is ridge ending and another, ridge bifurcation; they cannot be considered as a pair of corresponding minutiae and $R(i, j)=400$. Otherwise if $M(P)(i)$ and $M(Q)(j)$ are either ridge endings or ridge bifurcations, the value of $R(i, j)$ is defined below.

If the number of points recorded in the associate ridge of $M(P)(i)$ and $M(Q)(j)$ is not the same, $R(i, j)=400$. Otherwise let $R(P)$ represent the associated ridge of minutia $M(P)(i)$ and $R(Q)$ represent the associated ridge of minutia $M(Q)(j)$. $R(P)$ is matched against $R(Q)$ to get the difference of two ridges according to the following formula:

$$
\begin{aligned}
& d_{\text {Diff }}=\frac{1}{L} \sum_{n=1}^{L}\left|d\left(P, i_{n}\right)-d\left(Q, j_{n}\right)\right| \\
& \alpha_{\text {Diff }}=\frac{1}{L} \sum_{n=1}^{L}\left|\alpha\left(P, i_{n}\right)-\alpha\left(Q, i_{n}\right)\right|
\end{aligned}
$$

where $L(L \leqslant 4)$ is the number of points recorded.

If $d_{\text {Diff }} \leqslant T_{d}$ and $\alpha_{\text {Diff }} \leqslant T_{\alpha}\left(T_{d}\right.$ and $T_{\alpha}$ are empirical thresholds which changes with fingerprint images $P$ and $Q$ ), indicating that the form of the two ridges is similar to each other to a certain degree, minutiae $M(P)(i)$ and $M(Q)(j)$ can be considered as a pair of corresponding minutiae, and is calculated as:

$R(i, j)=\theta(P, i)-\theta(Q, j)$

where $\theta(P, i)$ is the orientation of $M(P)(i)$ and $\theta(Q, i)$ the orientation of $M(Q)(j)$.

If $d_{\text {Diff }} \leqslant T_{d}$ or $\alpha_{\text {Diff }} \geqslant T_{\alpha}$, indicating that the form of these two ridges is not similar to each other, minutiae $M(P)(i)$ and $M(Q)(j)$ cannot be considered as a pair of corresponding minutiae, and $R(i, j)=400$.

To align the input minutiae set with the template minutiae set in the polar coordinate system, it is necessary to convert the input minutiae and the template minutiae to the polar coordinate system with respect to the reference minutiae $M(P)(i)$ and $M(Q)(j)$, and add the rotation angle $R(i, j)$ to the radial angle of the polar coordinate of every input minutia to finish the alignment. 
Eq. (8) is used to convert other minutiae to the polar coordinate system with respect to the reference minutia.

$$
\left(\begin{array}{c}
\bar{r}_{i}^{s} \\
\bar{e}_{i}^{s} \\
\bar{\theta}_{i}^{s}
\end{array}\right)=\left(\begin{array}{c}
\sqrt{\left(x_{i}-x^{s}\right)^{2}+\left(y_{i}-y^{s}\right)^{2}} \\
\tan ^{-1}\left(\frac{y_{i}-y^{s}}{x_{i}-x^{s}}\right) \\
\theta_{i}-\theta^{s}
\end{array}\right)
$$

where $\left(x_{i}, y_{i}, \theta_{i}\right)^{\mathrm{T}}$ is the representation of a minutia in the Cartesian coordinate system. $\left(x^{s}, y^{s}, \theta^{s}\right)^{\mathrm{T}}$ represents the reference minutia in the Cartesian coordinate system. $\left(\bar{r}_{i}^{s}, \bar{e}_{i}^{s}, \bar{\theta}_{i}^{s}\right)^{\mathrm{T}}$ represents the minutia in the polar coordinate system where $\bar{r}_{i}^{s}$ indicates the radial distance, $\bar{e}_{i}^{s}$ the radial angle and $\bar{\theta}_{i}^{s}$ the orientation of the minutia, with respect to the reference minutia.

The aligned minutiae sets $M(P)$ and $M(Q)$ can be presented by $M\left(P^{i}\right)=\left\{M\left(P^{i}\right)(k)=\left\{\bar{r}_{k}^{i}, \bar{e}_{k}^{i}, \bar{\theta}_{k}^{i}\right.\right.$, $\left.\left.t_{k}, d_{k n} \alpha_{k n} ; n=1,2, \ldots, L\right\}^{\mathrm{T}} ; k=1,2, \ldots, m(P)\right\}$ and $M\left(Q^{j}\right)=\left\{M\left(Q^{j}\right)(l)=\left\{\bar{r}_{l}^{j}, \bar{e}_{l}^{j}, \bar{\theta}_{l}^{j}, t_{l}, d_{l n}, \alpha_{l n} ; n=1,2, \ldots\right.\right.$, $\left.L\}^{\mathrm{T}} ; l=1,2, \ldots, m(Q)\right\}$ respectively with their reference minutiae $M(P)(i)$ and $M(Q)(j)$.

\subsection{Setting of a variable sized bounding box}

To match between $M(P)$ and $M(Q)$, a fix-sized bounding box and a variable sized bounding box are introduced (Fig. 9).

The size of a bounding box is represented by $\alpha_{b}$ and $r_{b}$.

In the algorithm (Jain et al., 1997), a fix-sized bounding boxed is used, where $\alpha_{b}$ and $r_{b}$ assume (a)

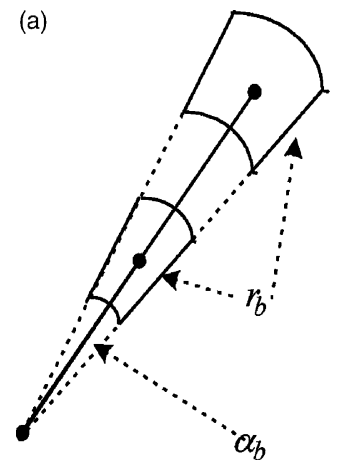

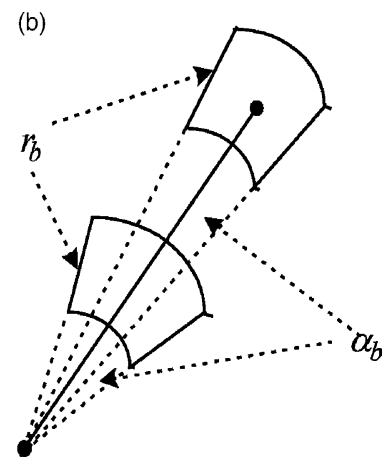

(b)
Fig. 9. (a) A fix-sized bounding box, (b) a variable sized bounding box. the same value for all the template minutiae. A variable sized bounding box is used with its values of $\alpha_{b}$ and $r_{b}$ in each minutia changed according to the radius of the minutia. If the radius of the template minutia is larger, its bounding box will have a larger $r_{b}$ and a smaller $\alpha_{b} . r_{b}$ and $\alpha_{b}$ are calculated as:

$r_{b}=\left\{\begin{array}{lll}r_{b s} & \text { if } \quad \frac{r}{a}<r_{b s} \\ \frac{r}{a} & \text { if } & r_{b s} \leqslant \frac{r}{a}<r_{b l} \\ r_{b l} & \text { if } & \frac{r}{a} \geqslant r_{b l}\end{array}\right.$

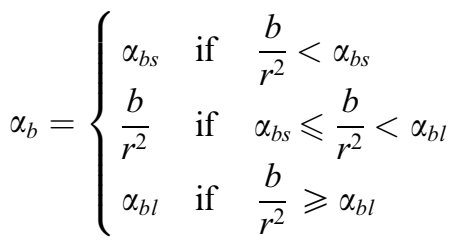

where $\alpha_{b s}, \alpha_{b l}, r_{b s}, r_{b l}$ are lower and upper bounds of the $r_{b}$ and $\alpha_{b}$ respectively. Parameters $a$ and $b$ are two predefined constants. $\alpha_{b s}, \alpha_{b l}, r_{b s}, r_{b l}, a$ and $b$ are empirical parameters which changes with the size of a fingerprint image.

To use a variable sized bounding box instead of a fix-sized one is to deal with non-linear deformation more robustly. When the radius of the minutia is small, a small deformation may cause a big change of the radial angle while the change of radius remains small. In this case, $\alpha_{b}$ of the bounding box should be larger and $r_{b}$ of the bounding box should be smaller. When the radius of the minutia is large, however, a small change in the radial angle may cause a big change in the position of the minutia. The radius can be largely deformed, as it is the accumulation of deformations from all the regions between the minutia and the reference minutia. Hence in this case $\alpha_{b}$ of the bounding box should be larger and $r_{b}$ of the box should be smaller.

\subsection{Aligned minutiae matching}

To match between $M(P)$ and $M(Q)$ is to identify whether two fingerprint images $P$ and $Q$ are from the same finger or not. The steps for matching 
between the two minutiae sets $M(P)$ and $M(Q)$ are described below:

Step 1. Selection of reference minutiae pair

For each minutia $M(P)(i)(1 \leqslant i \leqslant m(P))$ in the template minutiae set and each minutia $M(Q)(j)$ $(1 \leqslant j \leqslant m(Q))$ in the input minutiae set, if $R(i, j)=400$, indicating that $M(P)(i)$ and $M(Q)(j)$ can not be considered as a pair of corresponding minutiae, this step is repeated and another $M(P)(i)$ and $M(Q)(j)$ are chosen. Otherwise step 2 is taken. If all possible minutiae pairs have been considered, go to step 4.

Step 2. Alignment of two minutiae sets with their reference minutiae respectively

$M(P)(i)$ and $M(Q)(j)$ are taken as reference minutiae. Each minutia in $M(P)$ and $M(Q)$ is converted to the polar coordinate system with respect to the corresponding reference minutia with Eq. (8). Two minutiae sets $M(P)$ and $M(Q)$ are converted to:

$$
\begin{aligned}
M\left(P^{i}\right)=\left\{M\left(P^{i}\right)(k)=\left\{\bar{r}_{k}^{i}, \bar{e}_{k}^{i}, \bar{\theta}_{k}^{i}, t_{k}, d_{k n}, \alpha_{k n} ;\right.\right. \\
\left.n=1,2, \ldots, L\}^{\mathrm{T}} ; k=1,2, \ldots, m(P)\right\}
\end{aligned}
$$

and

$$
\begin{aligned}
M\left(Q^{j}\right)=\left\{M\left(Q^{j}\right)(l)=\left\{\bar{r}_{l}^{j}, \bar{e}_{l}^{j}, \bar{\theta}_{l}^{j}, t_{l}, d_{l n}, \alpha_{l n} ;\right.\right. \\
\left.n=1,2, \ldots, L\}^{\mathrm{T}} ; \quad l=1,2, \ldots, m(Q)\right\} .
\end{aligned}
$$

Step 3. Matching between $M\left(P^{i}\right)$ and $M\left(Q^{j}\right)$

$S(i, j)$ denotes the matching result of $M\left(P^{i}\right)$ and $M\left(Q^{j}\right), S(i, j)$ is calculated by

$S[i][j]=\sum_{k=1}^{M} \sum_{l=1}^{N} s_{k l}$

where $s_{k l}=1$ if $M\left(Q^{j}\right)(l)$ and $M\left(P^{i}\right)(k)$ satisfy the conditions that

(1) $M\left(Q^{j}\right)(l)$ is inside the bounding box of $M\left(P^{i}\right)(k)$. The bounding box of $M\left(P^{i}\right)(k)$ is decided with Eqs. (9), (10);

(2) The directional difference between $\bar{\theta}_{k}^{i}$ and $\bar{\theta}_{l}^{j}$ should be smaller than a predefined threshold value $\xi$ (empirically $\xi=15$ );

(3) $R(k, l) \neq 400$, which indicates that the associated ridges of $M\left(P^{i}\right)(k)$ and $M\left(Q^{j}\right)(l)$, should be similar to each other. else $s_{k l}=0$.

Then go to step 1;

Step 4. Output of the result of matching

$S_{r}$ denotes the matching score between $P$ and $Q$. It can be calculated by

$S_{r}=\max \{S(i, j)\}$

The higher the matching score $S_{r}$, the more likely $P$ and $Q$ are from the same finger. If $S_{r} \geqslant \varsigma$, $Q$ is considered to be from the same finger as $P$. Otherwise $P$ and $Q$ are considered to be from different fingers. $\varsigma$ is empirical threshold and is equal to 7 in this paper. $P$ and $Q$ may be statistically assumed to be from the same finger If the number of common minutiae between $M(P)$ and $M(Q)$ is greater than 6 .

During matching, the advantage of introducing ridge information embodied in $R(i, j)$ into the matching process lies in two aspects. Firstly, how to choose a reliable reference point pair is a very difficult problem in fingerprint matching. If all possible point pairs are considered, the pair that gives the largest matching score is chosen with a heavy burden. With the help of ridge information, the number of point pairs can be reduced to serve as the reference point pair. Each minutia $M(P)(i)$ $(1 \leqslant i \leqslant m(P))$ in $M(P)$ and each minutia $M(Q)(j)$ $(1 \leqslant j \leqslant m(Q))$ in $M(Q)$ can be used as the reference minutiae pair only when $R(i, j) \neq 400$, indicating that the associated ridges of $M(P)(i)$ and $M(Q)(j)$ are similar to each other in a certain degree. In this way, the problem in choosing the reference point pair is solved with low computational cost. Secondly, by introducing the ridge information into the matching process, our algorithm can better distinguish $P$ and $Q$ from different fingers while having little influence on the matching of the two images from the same finger. If $P$ and $Q$ come from the same finger, the associated ridges of their corresponding point pairs should be similar to each other to a certain degree. That is to say, $P$ and $Q$ are identified to be from the same finger, if $M(P)(k)$ and $M(Q)(l)$ are a corresponding point pair, it is likely $R(k, l) \neq 400$. But when $P$ and $Q$ are identified to be from different fingers, it is likely that $M(P)(k)$ and $M(Q)(l)$ satisfy all the other conditions of being a corresponding point pair but $R(k, l)=400$. 


\section{Experimental results}

In practice, the only public domain data sets are the US National Institute of Standards and Technology (NIST) CDROMs containing thousands of images scanned from paper cards where fingerprints were impressed by rolling "nail to nail" inked fingers. Since these images significantly differ from those acquired by optical or solid state sensors, they are not well suited for testing on "online" fingerprint systems, although they constitute an excellent benchmark for AFIS developments and fingerprint classification studies, NIST recently released a database containing digital videos of live-scanned fingerprint data. Since this database was specially collected for studying plastic distortion affecting the online acquisition process and the impact of finger rotation, it models only certain fingerprint variations and it is not recommendable for a general evaluation of verification algorithm (Maio et al., 2002). Thus we perform our tests using the fingerprint databases of FVC2000 to evaluate the performance of our algorithm and to be able to compare developments in fingerprint verification.

Experiments of both the improved algorithm and the original one are performed on the four databases of FVC2000 (Maio et al., 2002). The performance evaluation method in (Maio et al., 2002) is adopted in our experiments. Results are reported below.

C_R represents the improved matching algorithm, that is, the algorithm which uses a variable sized bounding box and introduces ridge information embedded in $R(i, j)$ in the matching process. C_NR represents the algorithm that uses a variable sized bounding box without applying ridge information in the matching process. F_R represents the algorithm that uses a fix-sized bounding box and apply ridge information in the matching process. F_NR represents the original algorithm (Jain et al., 1997), that is, the algorithm uses fixed a bounding box without applying ridge information in the matching process. All the other conditions of the four algorithms are the same. When a fix-sized bounding box is used, the size of the box is adjusted to the optimal size for each database. The definitions of equal error rate
(EER), false non-match rate (FNMR), false match rate (FMR), the value that EER would take if the matching failures were excluded from the computation of FMR and FNMR (EER*) and receiving operating curve (ROC) are defined in (Maio et al., 2002). All the experiments were conducted on Pentium III450 $\mathrm{MHz}$ machine running windows 2000. (Maio et al., 2002) shows that EER, EER*, the lowest FNMR at which no false matches occur (ZeroFMR), the lowest FMR at which no false non-matches occur (ZeroFNMR), and ROC can describe the performance of a fingerprint matching algorithm on a fingerprint database.

The experimental results (Tables 1-4) show that the performance of the improved algorithm is better than the original one over the four databases. Tables 1-4 and Figs. 10-13 describe the average performance over the four databases. Performance improvement is obtained by using a variable sized bounding box instead of the fix-sized

Table 1

Results of the four algorithms over DB1_a

\begin{tabular}{lllll}
\hline Method & $\begin{array}{l}\text { EER } \\
(\%)\end{array}$ & $\begin{array}{l}\text { EER* } \\
(\%)\end{array}$ & $\begin{array}{l}\text { ZeroFMR } \\
(\%)\end{array}$ & $\begin{array}{l}\text { ZeroFNMR } \\
(\%)\end{array}$ \\
\hline C_R & 3.85 & 3.85 & 12.11 & 99.52 \\
C_NR & 4.67 & 4.67 & 20.11 & 100 \\
F_R & 5.32 & 5.32 & 25.03 & 100 \\
F_NR & 5.59 & 5.59 & 24.14 & 100 \\
\hline
\end{tabular}

Table 2

Results of the four algorithms over DB2_a

\begin{tabular}{lllll}
\hline Method & $\begin{array}{l}\text { EER } \\
(\%)\end{array}$ & $\begin{array}{l}\text { EER* } \\
(\%)\end{array}$ & $\begin{array}{l}\text { ZeroFMR } \\
(\%)\end{array}$ & $\begin{array}{l}\text { ZeroFNMR } \\
(\%)\end{array}$ \\
\hline C_R & 1.77 & 1.77 & 5.46 & 100 \\
C_NR & 3.50 & 3.50 & 21.79 & 100 \\
F_R & 3.94 & 3.94 & 18.96 & 100 \\
F_NR & 4.25 & 4.25 & 24.46 & 100 \\
\hline
\end{tabular}

Table 3

Results of the four algorithms over DB3_a

\begin{tabular}{lccll}
\hline Method & $\begin{array}{l}\text { EER } \\
(\%)\end{array}$ & $\begin{array}{l}\text { EER* } \\
(\%)\end{array}$ & $\begin{array}{l}\text { ZeroFMR } \\
(\%)\end{array}$ & $\begin{array}{l}\text { ZeroFNMR } \\
(\%)\end{array}$ \\
\hline C_R & 9.25 & 9.25 & 22.04 & 100 \\
C_NR & 10.71 & 10.71 & 36.14 & 100 \\
F_R & 12.37 & 12.37 & 39.75 & 100 \\
F_NR & 11.26 & 11.26 & 44.64 & 100 \\
\hline
\end{tabular}


Table 4

Results of the four algorithms over DB4_a

\begin{tabular}{lllll}
\hline Method & $\begin{array}{l}\text { EER } \\
(\%)\end{array}$ & $\begin{array}{l}\text { EER* } \\
(\%)\end{array}$ & $\begin{array}{l}\text { ZeroFMR } \\
(\%)\end{array}$ & $\begin{array}{l}\text { ZeroFNMR } \\
(\%)\end{array}$ \\
\hline C_R & 5.67 & 5.67 & 25.5 & 100 \\
C_NR & 6.47 & 6.47 & 39.39 & 100 \\
F_R & 6.61 & 6.61 & 43.93 & 100 \\
F_NR & 6.96 & 6.96 & 56.71 & 100 \\
\hline
\end{tabular}

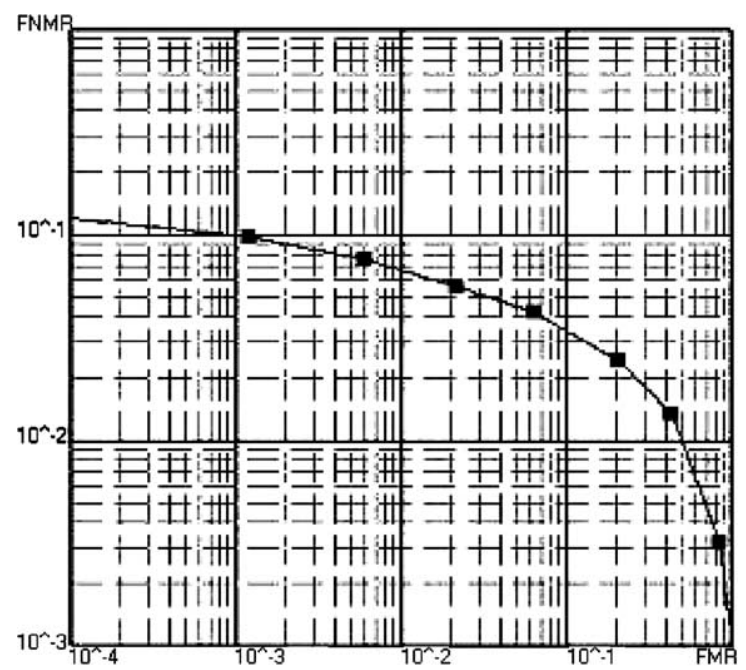

Fig. 10. The ROC curve of the improved algorithm over DB1_a.

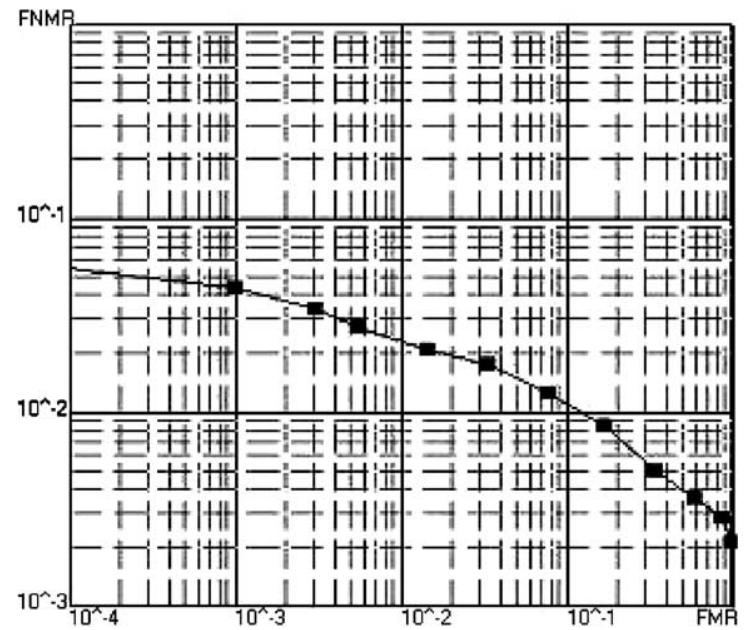

Fig. 11. The ROC curve of the improved algorithm over DB2_a.

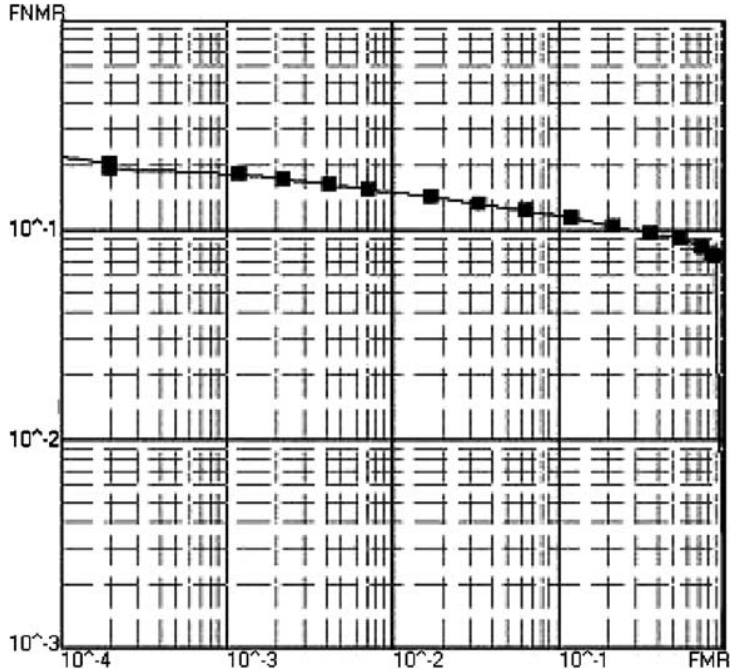

Fig. 12. The ROC curve of the improved algorithm over DB3_a.
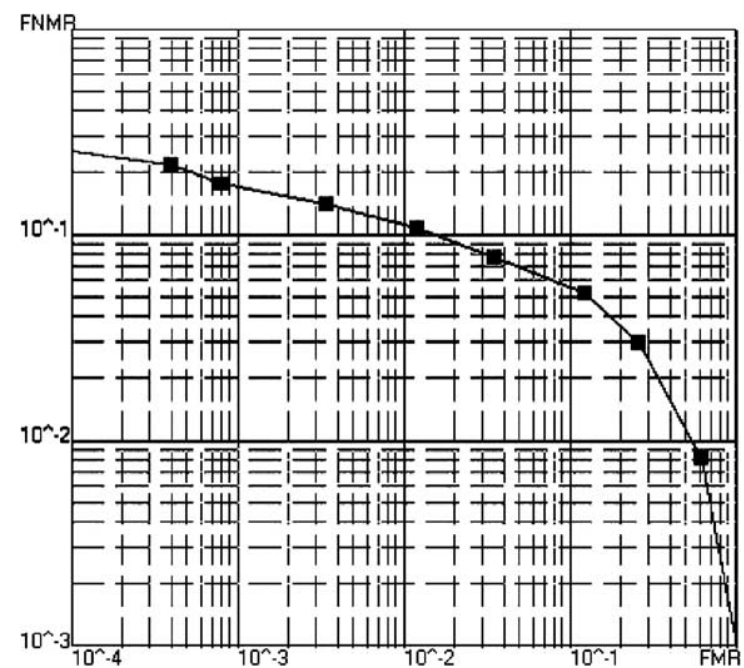

Fig. 13. The ROC curve of the improved algorithm over Db4_a.

one and by introducing ridge information into the matching process. The improvement of performance by introducing ridge information into matching is smaller and may even worsen the performance in some rare cases of matching with fix-sized bounding box overdatabase DB3_a (Table 3). By using both ridge information and variable sized bounding box, however, the perfor- 
mance of matching is better than those of other three matching algorithms on the four fingerprint databases of FVC2000. Furthermore, the average enrolling time and average matching time of our algorithm is less than those of other methods (Maio et al., 2002). Thus the statistical significance of the improvements prove that matching using both a variable sized bounding box method and ridge information is an effective and practical matching method.

\subsection{FVC2000 database DB1_a}

The average enrolling time and average matching time of C_R overDB1 is $0.52 \mathrm{~s}$.

\subsection{FVC2000 database DB2_a}

The average enrolling time and average matching time (Maio et al., 2002) of C_R overDB2 is $0.44 \mathrm{~s}$.

\subsection{FVC2000 database DB3_a}

The average enrolling time and average matching time (Maio et al., 2002) of C_R over DB3 is $1.06 \mathrm{~s}$.

\subsection{FVC2000 database DB4_a}

The average enrolling time and average matching time (Maio et al., 2002) of C_R over DB4 is $0.36 \mathrm{~s}$.

\section{Conclusion and discussion}

We have developed a fingerprint image enhancement method based on orientation field and introduced a minutiae matching algorithm, which is superior to the previously reported in (Jain et al., 1997), in which minutiae matching was done by point pattern matching in the polar coordinate system. In our method, simpler but more effective alignment is used where ridge information is introduced into the matching process apart from a variable sized bounding box in the matching process. These modifications make our method more robust to non-linear deformations between two fingerprint images.

Our experiments show that fingerprint images can be well matched using the minutiae matching method. But some fingerprint images of bad quality exist as well as unclear ridge structures that could not be matched by minutiae matching. The problem lies in the fact that in such images it is very difficult to extract minutiae correctly. Our future work will be along two aspects. First, the fingerprint image enhancement algorithm should be improved to extract minutiae more accurately so that the minutiae-matching algorithm is more robust to non-linear deformation (Cheng et al., 2002; Luo and Tian, 2000). Second, we try to find some new features of fingerprints, such as local features in frequency domain (Willis and Myers, 2001) and its local directional features in orientation fields (Coetzee and Botha, 1993; Lee and Wang, 2001), and to match without the minutiae feature for fingerprint images of bad quality. We believe it is better to use fingerprint-matching method that is not based on minutiae as a complementary way to the minutiae matching method in the system.

\section{Acknowledgements}

The authors would like to be grateful to the editors, anonymous reviewers and Prof. Qian Shouchu for their careful reading and helpful suggestions. This paper is supported by the National Natural Science Foundation of China under Grant Nos. 79990580, 69931010, 60172057, 60071002, 60072007; the National High Technology Development Program of China under Grant No. 2001AA144120.

\section{References}

Chang, S.-H., Cheng, F.-H., Hsu, W.-H., Wu, G.-Z., 1997. Fast algorithm for point pattern matching: Invariant to translations, rotations and scale changes. Pattern Recognit. 29, 311-316.

Cheng, J., Tian, J., Zhang, T., 2002. Fingerprint Enhancement with Dyadic Scale-Space, appeared to International Conference Pattern Recognition (ICPR, 2002). 
Coetzee, L., Botha, E.C., 1993. Fingerprint recognition in low quality images. Pattern Recognit. 26 (10), 1441-1460.

Farina, A., Kovacs-Vajna, Z.M., Leone, A., 1999. Fingerprint minutiae extraction from skeletonized binary images. Pattern Recognit. 32, 877-889.

Huang, D.C., 1993. Enhancement and feature purification of fingerprint images. Pattern Recognit. 26 (11), 1661-1671.

Jain, A., Lin, H., Bolle, R., 1997. On-line fingerprint verification. IEEE Trans. Pattern Anal. Machine Intell. 19 (4), 302-313.

Jiang, X., Yau, W.-Y., 2000. Fingerprint minutiae matching based on the local and global structures. In: Proc. 15th Internat. Conf. Pattern Recognition (ICPR, 2000) 2. pp. 1042-1045.

Lee, C.-J., Wang, S.-D., 2001. Fingerprint feature reduction by principal Garbor basis function. Pattern Recognit. 34 (11), 2245-2248.

Lin, H., Wan, Y., Jain, A., 1998. Fingerprint image enhancement: Algorithm and performance evaluation. IEEE Trans. Pattern Anal. Machine Intell. 20 (8), 777-789.

Luo, X., Tian, J., 2000. Knowledge based fingerprint image enhancement. In: Proc. 15th Internat. Conf. Pattern Recognition (ICPR, 2000) 4. pp. 783-786.
Maio, D., Maltoni, D., Cappelli, R., Wayman, J.L., Jain, A.K., 2002. FVC2000: Fingerprint Verification Competition. IEEE Trans. Pattern Anal. Machine Intell. 24 (3), 402-412.

Miklós, Z., Kovács-Vajna, Z.M., 2000. A fingerprint verification system based on triangular matching and dynamic time warping. IEEE Trans. Pattern Anal. Machine Intell. 22 (11).

Naccache, N.J., Shinghal, R., 1984. SPTA: A proposed algorithm for thinning binnary patterns. IEEE Trans. Systems Man Cybernet. SMC-14 (3), 409-418.

Ranade, S., Rosenfeld, A., 1980. Point pattern matching by relaxation. Pattern Recognit. 12, 269-275.

Ratha, N., Chen, S., Jain, A.K., 1995. Adaptive flow orientation based feature extraction in fingerprint images. Pattern Recognit. 28 (11), 1657-1672.

Xiao, Q., Raafat, H., 1991. Fingerprint image postprocessing: A combined statistical and structural approach. Pattern Recognit. 24 (10), 985-992.

Willis, A.J., Myers, L., 2001. A cost-effective fingerprint recognition systems for use with low-quality prints and damaged fingerprints. Pattern Recognit. 34 (2), 255270 . 